\title{
Electron cooling in diffusive normal metal-superconductor tunnel junctions with a spin-valve ferromagnetic interlayer
}

\author{
A. Ozaeta, ${ }^{1}$ A. S. Vasenko, ${ }^{2}$ F. W. J. Hekking, ${ }^{3}$ and F. S. Bergeret ${ }^{1,4}$ \\ ${ }^{1}$ Centro de Física de Materiales (CFM-MPC), Centro Mixto, Consejo Superior de Investigaciones Científicas - Universidad del País \\ Vasco/Euskal Herriko Unibertsitatea (CSIC-UPVIEHU), Manuel de Lardizabal 5, E-20018 San Sebastián, Spain \\ ${ }^{2}$ Institut Laue-Langevin, 6 rue Jules Horowitz, BP 156, F-38042 Grenoble, France \\ ${ }^{3}$ Laboratoire de Physique et Modélisation des Milieux Condensés, Université Joseph Fourier and CNRS, 25 Avenue des Martyrs, BP 166, \\ F-38042 Grenoble, France \\ ${ }^{4}$ Donostia International Physics Center (DIPC), Manuel de Lardizabal 4, E-20018 San Sebastián, Spain
}

(Received 30 March 2012; published 15 May 2012)

\begin{abstract}
We investigate heat and charge transport through a diffusive $\operatorname{SIF}_{1} \mathrm{~F}_{2} \mathrm{~N}$ tunnel junction, where $\mathrm{N}$ (S) is a normal (superconducting) electrode, $\mathrm{I}$ is an insulator layer, and $\mathrm{F}_{1,2}$ are two ferromagnets with arbitrary direction of magnetization. The flow of an electric current in such structures at subgap bias is accompanied by a heat transfer from the normal metal into the superconductor, which enables refrigeration of electrons in the normal metal. We demonstrate that the refrigeration efficiency depends on the strength of the ferromagnetic exchange field $h$ and the angle $\alpha$ between the magnetizations of the two F layers. As expected, for values of $h$ much larger than the superconducting order parameter $\Delta$, the proximity effect is suppressed and the efficiency of refrigeration increases with respect to a NIS junction. However, for $h \sim \Delta$ the cooling power (i.e., the heat flow out of the normal metal reservoir) has a nonmonotonic behavior as a function of $h$ showing a minimum at $h \approx \Delta$. We also determine the dependence of the cooling power on the lengths of the ferromagnetic layers, the bias voltage, the temperature, the transmission of the tunneling barrier, and the magnetization misalignment angle $\alpha$.
\end{abstract}

DOI: 10.1103/PhysRevB.85.174518

PACS number(s): 74.45.+c, 74.50.+r, 74.25.fc, 75.30.Et

\section{INTRODUCTION}

The presence of the superconducting energy gap leads to a selective tunneling of high-energy quasiparticles out of the normal metal in a normal metal-insulator-superconductor (NIS) tunnel junction. ${ }^{1,2}$ This phenomenon generates a heat current from the normal metal to the superconductor (also referred to as cooling power). The heat transfer through NIS junctions can be used for the realization of microcoolers. ${ }^{3-5}$ Present state-of-the-art experiments allow the reduction of the electron temperature in a normal metal lead from 300 to about $100 \mathrm{mK}$, offering perspectives for on-chip cooling of nanosized systems, such as high-sensitive detectors and quantum devices. ${ }^{6,7}$

The cooling power of tunnel junctions depends on several parameters, some of them controllable. For example the cooling power can be optimized by controlling the voltage across the junction. A maximized cooling effect is reached at a voltage bias just below the superconducting energy gap $\Delta$. Larger values of voltage, $e V \gtrsim \Delta$, lead to a larger charge current $I$ through the junction and hence to larger values of the Joule heating power (i.e., to a negative cooling power). A limitation of the performance of a NIS microcooler arises also from the fact that nonequilibrium quasiparticles injected into the superconducting electrode accumulate near the tunnel interface $^{7-9}$ As a consequence hot quasiparticles may tunnel back into the normal metal, leading to a reduction of the cooling effect. ${ }^{9,10}$ In order to overcome this problem a so-called quasiparticle trap, ${ }^{11}$ made of an additional normal metal layer has been attached to the superconducting electrode, removing hot quasiparticles from the superconductor. Recently, it was also shown that a small magnetic field enhances relaxation processes in a superconductor and leads to significant improvement of the cooling power in NIS junctions. ${ }^{12}$ Improved cooling performance can be also achieved by proper tuning of the tunneling resistances of the individual NIS tunnel junctions in a double junction SINIS cooling device. ${ }^{13}$

Another important limitation for NIS microcoolers arises from the intrinsic multiparticle nature of current transport in NIS junctions, which is governed not only by single-particle tunneling but also by two-particle processes due to the Andreev reflection. ${ }^{14}$ While the single-particle current and the associated heat current are due to quasiparticles with energies $E>\Delta$, at low temperatures or high junction transparencies the charge transport in NIS junctions is dominated by the Andreev reflection (i.e., by subgap processes). The Andreev current $I_{A}$ does not transfer heat through the NS interface but rather generates the Joule heating $I_{A} V$. At low enough temperatures this heating exceeds the single-particle cooling. ${ }^{15-17}$ The interplay between the single-particle tunneling and Andreev reflection sets a limiting temperature for the refrigeration $T_{\min }{ }^{17}$

One way to decrease $T_{\min }$ is to decrease the NIS junction transparency. However, large values of the contact resistance hinder carrier transfer and lead to a severe limitation in the achievable cooling powers. In order to increase the junction transparency and at the same time to reduce the Andreev current, it was suggested to use materials where the proximity effect is suppressed, such as ferromagnets, ferromagnetic insulators, and half metals. In particular Giazotto et al. studied theoretically a ballistic normal metal-ferromagnetsuperconductor structure within a phenomenological model 
and predicted an enhancement of the cooling efficiency compared to NIS junctions. ${ }^{18}$ The reason for that increase lies in the suppression of the Andreev reflection due to the band structure of the ferromagnetic metals. The electron involved in Andreev reflection and its time-reversed counterpart (hole) must belong to opposite spin bands; thus, suppression of the Andreev current occurs in a FS junction and its intensity depends on the degree of the electron polarization at the Fermi level which is proportional to the exchange field of the $\mathrm{F}$ layer. ${ }^{19-22}$ The enhancement of the cooling efficiency by the magneticfield-driven tunable suppression of the Andreev reflection in superconductor/two-dimensional electron gas nanostructures was also studied in Ref. 23. Note that theoretical studies of electron cooling in SF proximity systems were performed only in the ballistic case, ${ }^{18,24}$ while real metallic systems are in the diffusive limit. Moreover, ferromagnets show in general a multidomain structure that was not considered in previous articles.

In this work we present a quantitative analysis of the thermoelectric transport in NIS microcoolers with a diffusive ferromagnetic interlayer consisting of two magnetic domains with arbitrary direction of magnetization (so-called superconducting triplet spin-valve ${ }^{25}$ ). Based on the quasiclassical Keldysh Green functions formalism we compute the electric and heat currents through the junction. We show that the enhancement of the cooling power with respect to the NIS case, as proposed in Ref. 18, only works if the exchange field of the ferromagnetic interlayer $h$ is much larger than the superconducting order parameter $\Delta$. However, the cooling power shows a minimum value for $h \approx \Delta$. We also study the dependence of the cooling power on the angle $\alpha$ between the magnetizations of the ferromagnetic domains. In the case of weak ferromagnets (i.e., for $h \lesssim \Delta$ ) the antiparallel configuration $\alpha=\pi$ leads to higher values of the cooling power and smaller values of $T_{\min }$. By increasing $h$ this behavior is reversed and the monodomain configuration $(\alpha=0)$ is more favorable for the refrigeration. For large values of the exchange field, $h \gg \Delta$, the cooling power is almost independent on $\alpha$.

The paper is organized as follows. In the next section, we formulate the theoretical model and basic equations. In particular, we obtain the expressions for the electric and the heat current and identify the contributions corresponding to single-particle and Andreev tunneling events. In Sec. III we present and discuss the main results of our work. We finally conclude with a summary in Sec. IV.

\section{MODEL AND BASIC EQUATIONS}

We consider the $\mathrm{SIF}_{1} \mathrm{~F}_{2} \mathrm{~N}$ junction depicted in Fig. 1. A ferromagnetic bilayer $\mathrm{F}_{1} \mathrm{~F}_{2}$ of length $l_{12}=l_{1}+l_{2}$ smaller than the inelastic relaxation length ${ }^{26}$ is connected to a superconductor $(\mathrm{S})$ and a normal $(\mathrm{N})$ reservoir along the $x$ direction. The $\mathrm{F}_{1} \mathrm{~F}_{2}$ bilayer can either model a two-domain ferromagnet or an artificial hybrid magnetic structure. We consider the diffusive limit [i.e., the elastic scattering length $\left.\ell \ll \min \left(\xi_{h}, \xi\right)\right]$ where $\xi_{h}=\sqrt{\mathscr{D} / 2 h}$ is the characteristic penetration length of the superconducting condensate into the ferromagnet, $h$ is the value of the exchange field, $\xi=\sqrt{\mathscr{D} / 2 \Delta}$ is the superconducting coherence length and $\mathscr{D}$ is the diffusion coefficient (we set $\hbar=k_{B}=1$ and for simplicity we assume the same $\mathscr{D}$ in the whole structure).

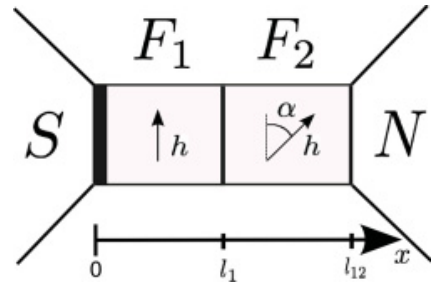

FIG. 1. (Color online) The $\operatorname{SIF}_{1} \mathrm{~F}_{2} \mathrm{~N}$ junction. The interface at $x=$ 0 corresponds to the insulating barrier (thick black line). Interfaces at $x=l_{1}$ and $x=l_{12}$ are fully transparent. $\alpha$ is the relative angle between the magnetization directions of $F_{1}$ and $F_{2}$.

We also assume that the $F_{1} F_{2}$ and $F_{2} N$ interfaces are transparent, while the $\mathrm{SF}_{1}$ is a tunnel interface. Thus, the two ferromagnetic layers are kept at the same potential as the voltage-biased normal reservoir. The magnetization of the $\mathrm{F}_{1}$ layer is along the $z$ direction, while the magnetization of the $F_{2}$ layer forms an angle $\alpha$ with the one of the layer $F_{1}$. Both magnetization vectors lie in the $y-z$ plane. Correspondingly, the exchange field vector in the $\mathrm{F}_{1}$ is given by $\mathbf{h}=(0,0, h)$, and in the $\mathrm{F}_{2}$ layer by $\mathbf{h}=(0, h \sin \alpha, h \cos \alpha)$, where the angle $\alpha$ takes values from 0 (parallel configuration) to $\pi$ (antiparallel configuration).

In order to describe the heat and electric currents through the structure we introduce the quasiclassical matrix Green function $\breve{G},{ }^{27,28}$

$$
\breve{G}=\left(\begin{array}{cc}
\check{G}^{R} & \check{G}^{K} \\
0 & \check{G}^{A}
\end{array}\right) .
$$

The latter is a matrix in the Keldysh $\times$ Nambu $\times$ spin space. The $R, A$, and $K$ indices stand for the retarded, advanced, and Keldysh components (we use the symbols. for $8 \times 8$ and . for $4 \times 4$ matrices). By neglecting nonequilibrium effects, the Keldysh component is related to the retarded and advanced ones by

$$
\begin{gathered}
\check{G}^{K}=\check{G}^{R} \check{n}-\check{n} \check{G}^{A}, \quad \check{n}=n_{+}+\tau_{z} n_{-}, \\
n_{ \pm}=\frac{1}{2}\left(\tanh \frac{E+e V}{2 T_{N}} \pm \tanh \frac{E-e V}{2 T_{N}}\right),
\end{gathered}
$$

where $n_{ \pm}$and $T_{N}$ are correspondingly the equilibrium quasiparticle distribution functions and the temperature in the normal reservoir and $\tau_{z}$ is the Pauli matrix in Nambu space. The retarded and advanced components are related via $\mathscr{G}^{A}=$ $-\tau_{z} \breve{G}^{R \dagger} \tau_{z} \cdot{ }^{27}$

The matrix Eq. (1) obeys the Usadel equation, ${ }^{29}$ which in the notations of Ref. 30 reads

$$
i \mathscr{D} \partial_{x} \breve{J}=\left[\tau_{z}(E-\mathbf{h} \sigma), \breve{G}\right], \quad \breve{J}=\breve{G} \partial_{x} \breve{G}, \quad \breve{G}^{2}=1,
$$

where $\sigma=\left(\sigma_{x}, \sigma_{y}, \sigma_{z}\right)$ are the Pauli matrices in spin space. In the $\mathrm{F}_{1}$ region $\mathbf{h} \sigma=h \sigma_{z}$ and the Usadel equation Eq. (3) has the form

$$
i \mathscr{D} \partial_{x} \breve{J}=\left[\tau_{z}\left(E-\sigma_{z} h\right), \breve{G}\right], \quad \breve{G}^{2}=1 .
$$

In the $\mathrm{F}_{2}$ region $\mathbf{h} \sigma=h \sigma_{z} \exp \left(-i \sigma_{x} \alpha\right)$. It is convenient to introduce Green's functions rotated in spin space, ${ }^{31}$

$$
\widetilde{G}=U^{\dagger} \breve{G} U, \quad U=\exp \left(i \sigma_{x} \alpha / 2\right) .
$$

The rotated function $\widetilde{G}$ is then determined by Eq. (4). 
The Usadel equation Eq. (4) should be complemented by boundary conditions at the interfaces. As mentioned above, we assume that the $\mathrm{F}_{1} \mathrm{~F}_{2}$ and $\mathrm{F}_{2} \mathrm{~N}$ interfaces are transparent and therefore the boundary conditions at $x=l_{1}, l_{12}$ read

$$
\begin{gathered}
\left.\breve{G}\right|_{x=l_{1}-0}=\left.\breve{G}\right|_{x=l_{1}+0}, \\
\left.\partial_{x} \breve{G}\right|_{x=l_{1}-0}=\left.\partial_{x} \breve{G}\right|_{x=l_{1}+0}, \\
\left.\breve{G}\right|_{x=l_{12}-0}=\tau_{z} .
\end{gathered}
$$

At $x=0$, the $\mathrm{SF}_{1}$ interface is a tunnel barrier, and we may use the Kupriyanov-Lukichev boundary conditions, ${ }^{32}$

$$
\left.\breve{J}\right|_{x=0}=(W / \xi)\left[\breve{G}_{S}, \breve{G}\right]_{x=0},
$$

where $\breve{G}_{S}$ is the Green function of a bulk BCS superconductor defined as

$$
\begin{aligned}
\breve{G}_{S} & =\tau_{z} u+\tau_{x} v, \\
(u, v) & =(E, i \Delta) / \epsilon, \quad \epsilon=\sqrt{(E+i \eta)^{2}-\Delta^{2}},
\end{aligned}
$$

where $\eta$ describes inelastic scattering rate and $W \ll 1$ is the diffusive transparency parameter, ${ }^{33} W=\xi / 2 g_{N} R$. Notice that $W$ is temperature dependent, since the coherence length $\xi$ is proportional to $\Delta^{-1 / 2}(T)$. In Sec. III we set $\eta \simeq 10^{-3} \Delta_{0}$ in our calculations, where $\Delta_{0}$ is the superconducting gap at $T=0$. In the following we omit $\eta$ in our analytical expressions for simplicity.

Because of the low transparency of the $\mathrm{SF}_{1}$ barrier, the proximity effect is weak and the retarded Green function can be linearized (we omit the superscript $R$ ),

$$
\check{G} \approx \tau_{z}+\tau_{x} \hat{f},
$$

where $\hat{f}$ is the $2 \times 2$ anomalous Green function in the spin space $(|\hat{f}| \ll 1)$ that obeys the linearized Usadel equation,

$$
i \mathscr{D} \partial_{x x}^{2} \hat{f}=2 E \hat{f}-\{\mathbf{h} \sigma, \hat{f}\},
$$

where $\{\cdot, \cdot\}$ stands for the anticommutator.

The general solution of Eq. (12) has the form

$$
\hat{f}(x)=f(x)+f_{y}(x) \sigma_{y}+f_{z}(x) \sigma_{z},
$$

where $f$ is the singlet component and $f_{z}, f_{y}$ are the triplet components with respectively zero and \pm 1 projections on the spin quantization axis (we choose the $z$ axis). The $f_{y}$ term is usually known as the long-range triplet component because it describes Cooper pairs with parallel spins which survive the strong exchange splitting and can diffuse into the ferromagnet over larger distances compared to the singlet component. ${ }^{34-38}$ Indeed, by substituting Eq. (13) into Eq. (12) and using the boundary conditions Eqs. (6)-(9) one can compute the components $f(x), f_{y}(x)$, and $f_{z}(x)$, and easily show that while $f(x)$ and $f_{z}(x)$ decay into the ferromagnet over the magnetic length $\sqrt{\mathscr{D} / 2 h}$ the long-range component $f_{y}(x)$ decays over the length given by $\sqrt{\mathscr{D} / 2 E}$.

The charge and energy currents, $I$ and $Q$ respectively, can be obtained from ${ }^{27,28,39,40}$

$$
\begin{aligned}
I & =\frac{g_{N}}{e} \int_{0}^{\infty} I_{-} d E, \quad Q=\frac{g_{N}}{e^{2}} \int_{0}^{\infty} E I_{+} d E, \\
I_{-} & \equiv(1 / 8) \operatorname{Tr} \tau_{\mathrm{z}} \breve{\mathrm{J}}^{\mathrm{K}}, \quad \mathrm{I}_{+} \equiv(1 / 8) \operatorname{Tr} \tau_{0} \breve{\mathrm{J}}^{\mathrm{K}}, \\
\check{J}^{K} & \equiv\left(\breve{G} \partial_{x} \breve{G}\right)^{K}=\check{G}^{R} \partial_{x} \check{G}^{K}+\check{G}^{K} \partial_{x} \check{G}^{A},
\end{aligned}
$$

where $\breve{J}^{K}$ is the Keldysh component of the matrix current defined in Eq. (3) and $\tau_{0}$ is the unitary matrix in Nambu space.

From Eqs. (9), (10a), and (13) we determine both currents, $I$ and $Q$ at the $\mathrm{SF}_{1}$ interface

$$
\begin{aligned}
I & =\frac{1}{e R} \int_{0}^{\infty} n_{-}\left(\operatorname{Re} u+\operatorname{Re} v \operatorname{Re} f_{0}\right) d E \\
Q & =\frac{1}{e^{2} R} \int_{0}^{\infty} E\left(n_{+}-n\right)\left(\operatorname{Re} u-\operatorname{Im} v \operatorname{Im} f_{0}\right) d E
\end{aligned}
$$

where $n=\tanh \left(E / 2 T_{S}\right)$ and $T_{S}$ are the equilibrium quasiparticle distribution function and temperature in the superconducting reservoir respectively. The function $\left.f_{0} \equiv f\right|_{x=0}$ is the singlet component of $\hat{f}$ at $x=0$. Notice that only the singlet component of $\hat{f}$ enters the equations for the electric and energy currents. There is however an indirect dependence of the currents on the triplet component since the amplitude of $f_{0}$ in turn depends on the amplitudes of the triplet $f_{y}$ and $f_{z}$.

We now examine Eqs. (15) and discuss separately two main contributions for the currents $I$ and $Q$, which originate from the single-particle $(E>\Delta)$ and Andreev $(0<E<\Delta)$ processes. Let us focus first on the single-particle contributions $I_{1}$ and $Q_{1}$. For energies larger than the superconducting gap $(E>\Delta)$ only the terms proportional to $\operatorname{Re} u$ in Eqs. (15) are nonzero. From Eq. (10b) and Eq. (15a) we obtain the single-particle contribution to the electric current

$$
I_{1}=\frac{1}{e R} \int_{\Delta}^{\infty} N_{S}(E) n_{-}(E) d E,
$$

where $N_{S}(E)=|E| \Theta(|E|-\Delta) / \sqrt{E^{2}-\Delta^{2}}$ is the BCS normalized density of states (DOS) and $\Theta(x)$ is the Heaviside step function.

Rewriting in Eq. (16) the $n_{-}(E)$ function in terms of the Fermi function in the $\mathrm{N}$ reservoir, $n_{F}(E)=[1+$ $\left.\exp \left(E / T_{N}\right)\right]^{-1}$, we arrive at the well known expression for the tunneling current, ${ }^{41}$

$$
I_{1}=\frac{1}{e R} \int_{-\infty}^{\infty} N_{S}(E)\left[n_{F}(E-e V)-n_{F}(E)\right] d E .
$$

Note that within the linear approach the normalized DOS in the F layer is equal to unity and therefore the single particle electric current is independent of $f_{0}$.

The single-particle contribution to the energy current can be obtained from Eq. (15b),

$$
Q_{1}=\frac{1}{e^{2} R} \int_{\Delta}^{\infty} E\left(n_{+}-n\right)\left[N_{S}(E)-M_{S}^{+}(E) \operatorname{Im} f_{0}\right] d E,
$$

where $M_{S}^{+}(E)=\Delta \Theta(|E|-\Delta) / \sqrt{E^{2}-\Delta^{2}}$.

For energies $E<\Delta$ the electric charge is transferred by means of the Andreev reflection. The subgap current or Andreev current can be obtained from Eq. (15a),

$$
I_{A}=\frac{1}{e R} \int_{0}^{\Delta} n_{-}(E) M_{S}^{-}(E) \operatorname{Re} f_{0} d E .
$$

where $M_{S}^{-}(E)=\Delta \Theta(\Delta-|E|) / \sqrt{\Delta^{2}-E^{2}} \cdot{ }^{42}$

According to Eq. (15b) the contribution of the Andreev processes to the energy current vanishes, $Q_{A}=0$. 
We are interested here in the cooling power $P$ (i.e., in the heat current flowing out of the normal metal reservoir). One can express the cooling power in terms of the contributions introduced previously, ${ }^{17}$

$$
P=-Q-I V=P_{1}+P_{A},
$$

where

$$
P_{1}=-Q_{1}-I_{1} V, \quad P_{A}=-I_{A} V .
$$

From Eqs. (17)-(19) it is clear that for equal temperature of the electrodes $\left(T_{N}=T_{S}\right)$ and no bias voltage the cooling power vanishes. For a finite voltage $V$, on one hand the heat is taken from the $\mathrm{N}$ reservoir and is released in the superconductor. On the other hand there is a global heat production in both electrodes due to the Joule heating. In particular the Andreev current $I_{A}$ contributes to the Joule heating $I_{A} V$, which is fully released in the normal metal electrode and leads to a reduction of the cooling power.

In the next section we calculate the cooling power of the $\mathrm{SIF}_{1} \mathrm{~F}_{2} \mathrm{~N}$ junction as a function of the different parameters by solving Eqs. (17)-(20).

\section{RESULTS AND DISCUSSION}

According to Eqs. (15), in the linear case both the electric and heat currents are determined by the singlet component $f_{0}$ of the anomalous Green function, Eq. (13), evaluated at the $\mathrm{SF}_{1}$ interface $(x=0)$. Solving Eq. (12) in the $\mathrm{F}_{1}$ layer we obtain for the components of Eq. (13),

$$
\begin{aligned}
& f_{ \pm}(x)=a_{ \pm} \cosh \left(k_{ \pm} x\right)+\frac{2 W}{k_{ \pm}}\left(u a_{ \pm}-v\right) \sinh \left(k_{ \pm} x\right), \\
& f_{y}(x)=a_{y} \cosh \left(k_{y} x\right)+\frac{2 W}{k_{y}} u a_{y} \sinh \left(k_{y} x\right),
\end{aligned}
$$

where $f_{ \pm}=f \pm f_{z}, a_{i}$ are the boundary values of $f_{i}$ at $x=0$ ( $i$ stands for,,$+- y$ ) and

$$
k_{ \pm}=\sqrt{\frac{2(E \mp h)}{i \mathscr{D}}}, \quad k_{y}=\sqrt{\frac{2 E}{i \mathscr{D}}} .
$$

In the $\mathrm{F}_{2}$ layer the general solution has the form,

$$
\widetilde{f_{i}}(x)=b_{i} \sinh \left[k_{i}\left(x-l_{12}\right)\right],
$$

where $\tilde{f}_{i}$ are the components of the rotated Green function, Eq. (5).

Using the boundary conditions at the $F_{1} F_{2}$ interface, Eqs. (6) and (7) one obtain a set of six linear equations for the six coefficients $a_{i}$ and $b_{i}$, that can be solved straightforwardly. In particular we are interested in the value of the singlet component of the anomalous Green function at $x=0$, which is given by $f_{0}=\left(a_{+}+a_{-}\right) / 2$. The analytical expression has a lengthy, awkward form and we do not present it here. Once we obtain $f_{0}$ we compute the charge and energy currents from Eqs. (16), (18), and (19). Finally, using Eq. (20) we determine the cooling power. In what follows we assume that the temperatures of the $\mathrm{S}$ and $\mathrm{N}$ reservoirs to be equal, $T_{S}=T_{N}=T$, and neglect nonequilibrium effects in the ferromagnetic interlayer. ${ }^{17}$

The bias voltage between the $\mathrm{S}$ and $\mathrm{N}$ reservoirs is an easily adjustable experimental parameter, so all our curves except those presented in Fig. 5 are calculated for optimal value of the voltage bias $V_{\mathrm{opt}}$, at which the cooling power reaches its maximum for given values of the other parameters. In what follows, we assume the quantity $W$ to be taken at $T=0$, allowing for its temperature dependence in Eqs. (22) by means of corresponding temperature-dependent factors. In the subsequent analysis the cooling power $P$ is given in units of $\Delta_{0}^{2} / e^{2} R_{0}$, where $\Delta_{0}$ is the value of $\Delta$ at zero temperature and $R_{0}$ is the junction resistance at a fixed value $W=10^{-2}$ of the tunneling parameter.

We first study the dependence of the cooling power on the strength of the exchange field $h$. This dependence is shown in Fig. 2 for three different angles $\alpha=0, \pi / 2, \pi$ between the magnetizations of $F_{1}$ and $F_{2}$ layers at the optimum value of bias voltage. We have chosen the values of the temperature and tunneling parameter $W$ such that the Andreev current role in the cooling processes is essential (see Fig. 6). ${ }^{17}$ The thickness of the $\mathrm{F}$ layers is chosen to be $l_{1}=\xi$ and $l_{2}=6 \xi$.

Depending on the value of $l_{1} / \xi_{h}$, where $\xi_{h}=\sqrt{\mathscr{D} / 2 h}$ is the characteristic penetration length of the superconducting condensate into $\mathrm{F}_{1}$, one identifies different behaviors. If $l_{1} \gg \xi_{h}$ [i.e. for large values of $h / \Delta(T)$ ] the amplitude of the superconducting condensate in $\mathrm{F}_{2}$ can be neglected, as well as the dependence of the $f_{0}$ function on the angle $\alpha$. Thus, in the limit $h / \Delta(T) \gg 1$, the value of the cooling power does not depend on $\alpha$. Moreover, this asymptotic value is larger than in the nonmagnetic case $(h=0)$. This is a consequence of the strong suppression of the singlet correlations in $F_{1}$ due to the exchange field and hence of the Joule heating associated to the Andreev current [see Eq. (20)]. Note that for the value of temperature used in our figures $\Delta(T) \approx \Delta_{0}$.

In the opposite limit, $l_{1} / \xi_{h} \ll 1$, the characteristic penetration length depends weakly on $h$, and therefore the cooling power is also $\alpha$ independent. However, by increasing $h$ the cooling power first decreases and reaches a minimum. This unexpected behavior is qualitatively similar for all magnetic configurations and is a consequence of the Andreev current

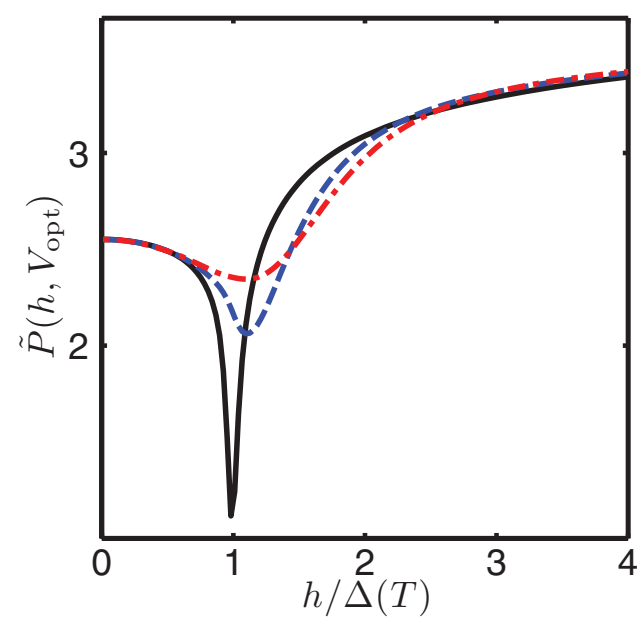

FIG. 2. (Color online) Cooling power versus exchange field for different orientations of the exchange field vector in the second ferromagnetic layer $\mathrm{F}_{2}: \alpha=0$ (black solid line), $\alpha=\pi / 2$ (blue dashed line), and $\alpha=\pi$ (red dash-dotted line), calculated at optimum bias; $W=7 \times 10^{-3}, T=0.25 \Delta_{0}, l_{1}=\xi$, and $l_{2}=6 \xi$. We have defined $\tilde{P}=10^{2} P\left(V_{\text {opt }}\right) e^{2} R_{0} / \Delta_{0}^{2}$. 

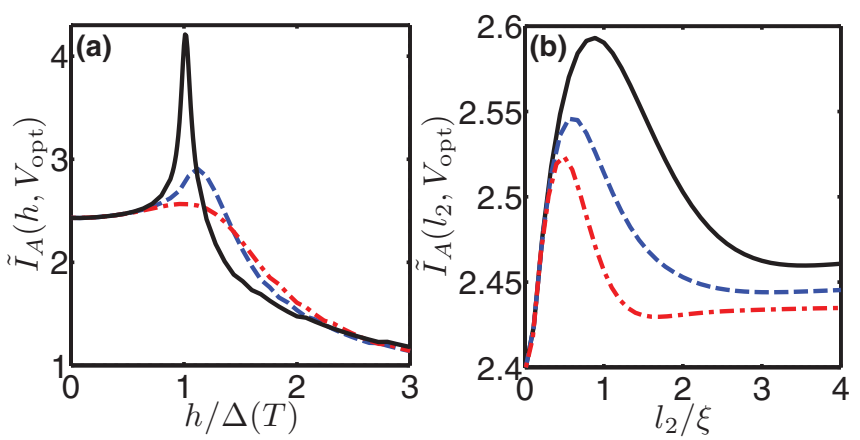

FIG. 3. (Color online) The Andreev current as a function of (a) the exchange field for $l_{2}=6 \xi$ and as a function of (b) the $\mathrm{F}_{2}$ length for $h=0.7 \Delta(T)$. Different magnetic configurations are chosen: $\alpha=0$ (solid black line), $\alpha=\pi / 2$ (dashed blue line), $\alpha=\pi$ (dash-dotted red line). The Andreev current is calculated at optimal bias; $W=7 \times 10^{-3}, T=0.25 \Delta_{0}, l_{1}=\xi$. We have defined $\tilde{I}_{A}=$ $I_{A}\left(V_{\mathrm{opt}}\right) e R_{0} / \Delta_{0}$

peak at $h \approx \Delta(T)$ (for monodomain case) in the finite temperature and finite voltage regime [see Fig. 3(a)] solid black line. However, there are quantitative differences between the monodomain ( $\alpha=0)$ and two-domain $(\alpha=\pi, \pi / 2)$ configurations. For $\alpha=0, P(h)$ shows a minimum at $h \approx \Delta(T)$. It is worth mentioning that around this minimum the cooling power of the $\mathrm{SIF}_{1} \mathrm{~F}_{2} \mathrm{~N}$ system is lower than that of the NIS junction $(h=0)$. By increasing the angle $\alpha$ the minimum is less pronounced and shifts to larger values of $h \gtrsim \Delta(T)$. For these values of $h$ and for $l_{1}=\xi$ the superconducting condensate can penetrate both ferromagnetic layers. Thus, the effective exchange field $\bar{h}$ acting on the Cooper pairs is a field, averaged over the length $\xi_{h} .{ }^{43}$ The $\bar{h}(\alpha)$ is gradually reduced as $\alpha$ increase from 0 to $\pi$. As before the cooling power minimum is at $\bar{h}(\alpha) \approx \Delta(T)$, which in the case of a finite $\alpha$ corresponds to larger values of the bare $h$. The minimum of the cooling power (Fig. 2), corresponds to a maximum of the Andreev current [Fig. 3(a)]. The unexpected nonmonotonic behavior of the Andreev current at small exchange fields $h \sim \Delta(T)$ is due to the competition between two-particle tunneling processes and decoherence mechanisms as quantitatively explained in a recent work by the authors. ${ }^{44}$

We analyze now the dependence of the cooling power on the length of the ferromagnetic bridge $F_{1} F_{2}$. To do this, we fix the thickness of $F_{1}$ at $l_{1}=\xi$ and vary $l_{2}$. Figure 4 shows the $P\left(l_{2}\right)$ dependence for two different values of the exchange field $h / \Delta(T)=0.7,1.7$ and different magnetic configurations $\alpha=$ $0, \pi / 2, \pi$. As expected all curves tend to a finite asymptotic value when $l_{2} \gg \xi$. This value however depends on $\alpha$.

In the case of an exchange field smaller than the superconducting gap $[h=0.7 \Delta(T)$, see Fig. 4(a)] the cooling power first reduces monotonically to a minimum by increasing $l_{2}$, then enhances to a maximum and finally reduces to the asymptotic value. Such behavior is preserved for all magnetic configurations and it follows from the nonmonotonic behavior of the Andreev current, shown in Fig. 3(b). Decrease of the Andreev current corresponds to the increase of the cooling power and vice versa. As shown in Fig. 3(b), at large values of $l_{2}$ the Andreev current increases by decreasing $l_{2}$, reaches a maximum and finally decreases for $l_{2} \lesssim \xi$. The strong

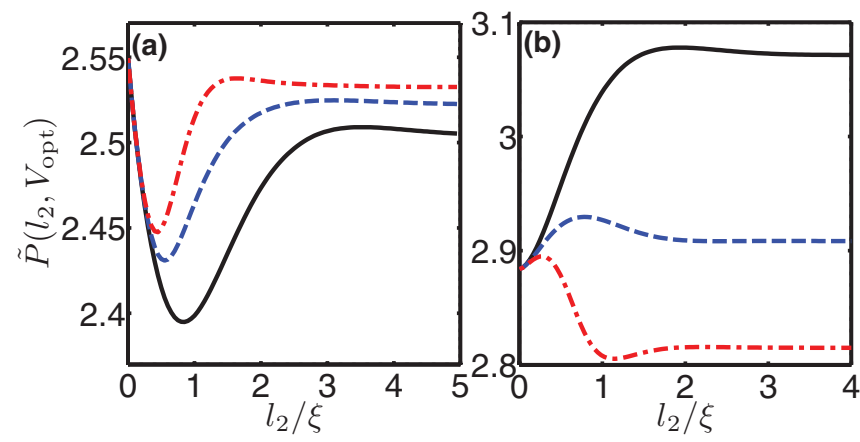

FIG. 4. (Color online) Cooling power versus length $l_{2}$ of the $\mathrm{F}_{2}$ layer for (a) $h=0.7 \Delta(T)$ and (b) $h=1.7 \Delta(T)$. We consider different orientations of the exchange field vector in the second ferromagnetic layer $F_{2}$ with respect to the one in $F_{1}: \alpha=0$ (solid black line), $\alpha=\pi / 2$ (dashed blue line), $\alpha=\pi$ (dash-dotted red line), and calculate the cooling power at optimal bias; $W=7 \times 10^{-3}$, $T=0.25 \Delta_{0}$, and $l_{1}=\xi . \tilde{P}$ is defined in Fig. 2 .

suppression of the Andreev current for small values of $l_{2}$ is due to the proximity of the $\mathrm{N}$ reservoir at $x=l_{12}$. On the other hand for larger values of $l_{2}$ the superconducting proximity effect in the ferromagnetic bridge is fully developed and leads to an increase of the Andreev current. It is remarkable that the cooling power for $\alpha=\pi$ is larger than the one at $\alpha=0$ for all values of $l_{2}$. In this case a lower effective exchange field $\bar{h}$ leads to larger values of the cooling power, due to the shift of the minimum of $P(h)$ observed in Fig. 2.

For an exchange field larger than $\Delta(T)[h=1.7 \Delta(T)$, see Fig. 4(b)] the behavior of the cooling power as a function of $l_{2}$ strongly depends on $\alpha$. For a monodomain magnet, $\alpha=0$, the cooling power increases monotonically by increasing $l_{2}$ until it reaches the asymptotic value due to the suppression of the Andreev current as in the ballistic case studied in Ref. 18. Similarly, in the antiparallel configuration $(\alpha=\pi)$, the cooling power first increases by increasing $l_{2}$, however for a larger value of $l_{2}$ reaches a maximum and then decreases. The presence of $F_{2}$ with a magnetization antiparallel to the one of $F_{1}$ leads to a reduced effective exchange field of the $\mathrm{F}_{1} \mathrm{~F}_{2}$ bridge. Thus, the Andreev current contribution is enhanced with respect to the one in the case $l_{2}=0$. As intuitively expected the cooling power (Andreev current) reaches a minimum (maximum) when $l_{2} \sim l_{1}=\xi$ (i.e., when the average magnetization is minimized). Further increase of $l_{2}>\xi$ leads to a suppression of the Andreev current and therefore to an increase of $P$ until the asymptotic values are reached. Figure 4(b) also shows the intermediate case $\alpha=\pi / 2$.

We now analyze the dependence of the cooling power on the bias voltage $e V$, tunneling parameter $W$, and temperature $T$. In our subsequent analysis we consider three different values of the exchange field $h=\Delta(T), 1.7 \Delta(T), 8 \Delta(T)$, and three magnetic configurations $\alpha=0, \pi / 2, \pi$. We set $l_{1}=\xi$, short enough for the pair correlation to be substantial in the $F_{2}$ layer [for $h=\Delta(T), 1.7 \Delta(T)$ ] and $l_{2}=6 \xi$, long enough to ensure the asymptotic regime (see Fig. 4). Figures 5, 6, and 7 show the cooling power as a function of $e V, W$, and $T$. A common feature of these figures is that the range of values of $V, W$, and $T$, for which the cooling power is positive increases 

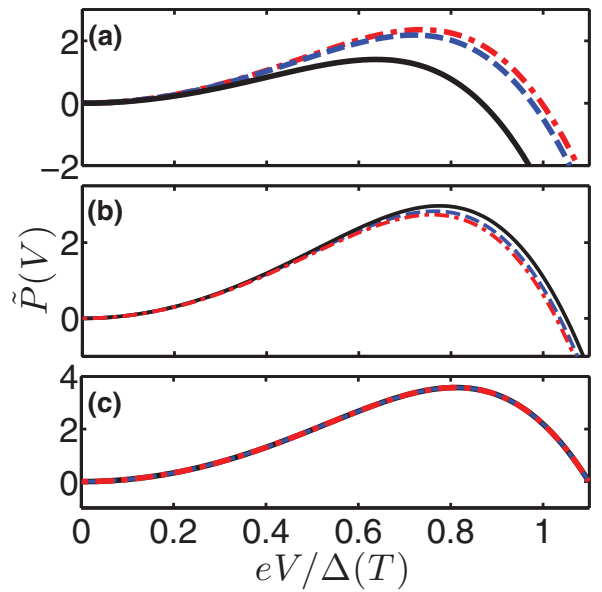

FIG. 5. (Color online) Cooling power versus bias voltage for $h=\Delta(T)$ (a), $h=1.7 \Delta(T)$ (b), and $h=8 \Delta(T)$ (c) for different orientations of the exchange field vector in the second ferromagnetic layer $\mathrm{F}_{2}: \alpha=0$ (black solid line), $\alpha=\pi / 2$ (blue dashed line), and $\alpha=\pi$ (red dash-dotted line); $W=7 \times 10^{-3}, T=0.25 \Delta_{0}, l_{1}=\xi$, and $l_{2}=6 \xi . \tilde{P}$ is defined in Fig. 2 .

by increasing $h$. Also the magnitude of the cooling power increases with $h$. This is in agreement with the qualitative predictions of Ref. 18. Note that the shape of all curves in Figs. 5, 6, and 7 does not depend significantly on the angle $\alpha$.

Figures 5 and 6 show that for low values of $e V$ and $W$, respectively, the cooling power depends only weakly on the relative magnetization angle $\alpha$. However, by increasing $\mathrm{eV}$ and $W$ the difference becomes appreciable, in particular for $h \approx \Delta(T)$.

As shown in Fig. 5 at certain value of $e V_{\mathrm{opt}} \lesssim 0.8 \Delta(T)$, the cooling power reaches its maximum value $P_{\max }=P\left(V_{\text {opt }}\right)$. The $e V_{\text {opt }}$ value is the one used as optimal bias value in the figures. For voltages larger than this optimal value, the

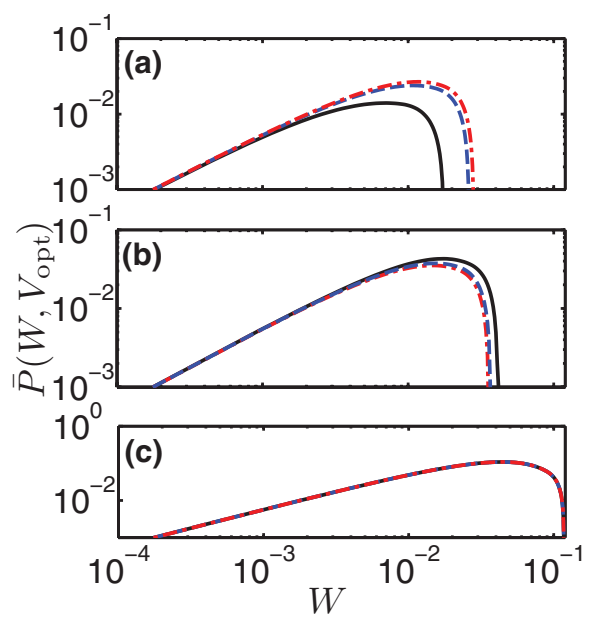

FIG. 6. (Color online) Dependence of the cooling power on the tunneling parameter $W$ for $h=\Delta(T)(\mathrm{a}), h=1.7 \Delta(T)(\mathrm{b})$, and $h=$ $8 \Delta(T)(\mathrm{c})$ and for different orientations of the exchange field vector in the second ferromagnetic layer $\mathrm{F}_{2}: \alpha=0$ (black solid line), $\alpha=\pi / 2$ (blue dashed line), and $\alpha=\pi$ (red dash-dotted line). $P$ is calculated at optimum bias; $T=0.25 \Delta_{0}, l_{1}=\xi$, and $l_{2}=6 \xi$. We have defined $\bar{P}\left(W, V_{\text {opt }}\right)=P\left(W, V_{\text {opt }}\right) e^{2} R_{0} / \Delta_{0}^{2}$. Note the logarithmic scale.

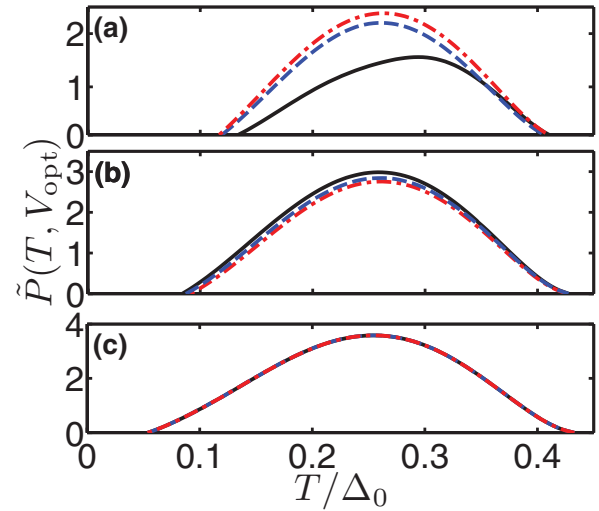

FIG. 7. (Color online) Temperature dependence of the cooling power for $h=\Delta_{0}$ (a), $h=1.7 \Delta_{0}$ (b), and $h=8 \Delta_{0}$ (c), and for different orientations of the exchange field vector in the second ferromagnetic layer $\mathrm{F}_{2}: \alpha=0$ (black solid line), $\alpha=\pi / 2$ (blue dashed line), and $\alpha=\pi$ (red dash-dotted line). $P$ is calculated at optimum bias; $W=7 \times 10^{-3}, l_{1}=\xi$, and $l_{2}=6 \xi$. $\tilde{P}$ is defined in Fig. 2.

quasiparticle current $I$ and hence the Joule heating power $I V$ increases drastically leading to a rapid decrease of the cooling power. As can be seen from Figs. 5, 6, and 7 the optimal voltage $V_{\text {opt }}$ depends on the temperature $T$, tunneling parameter $W$, and magnetic configuration angle $\alpha$. For the exchange field equal to the superconducting gap the maximal cooling power $P_{\max }$ is largest in the antiparallel configuration, while for larger $h=1.7 \Delta(T)$ the largest value $P_{\max }$ is in the parallel configuration, in agreement with Fig. 2.

Figure 6 shows that the cooling power has also a maximum as a function of $W$. Increasing $W$ the cooling power first linearly increases as single electron tunneling dominates. For larger values of the tunneling parameter, the Andreev current heating dominates over the single-particle cooling and leads to a rapid decrease of the cooling power, which tends to zero at a certain onset point. As the exchange field increases, the role of Andreev processes becomes less important, therefore the onset shifts toward larger values of $W$. This means that for higher exchange field in the ferromagnetic interlayer one may use weaker tunnel barriers for the microcooler fabrication, which leads to higher amplitudes of the cooling power [see Fig. 6(c)] and more effective electron refrigeration.

In Fig. 7 we show the temperature dependence of the cooling power. At $T \gtrsim 0.42 \Delta_{0} \approx 0.75 T_{c}$, where $T_{c}$ is the critical temperature of the superconductor, the cooling power becomes negative for all voltages. This value of the temperature holds for a wide range of parameters. ${ }^{17}$ The existence of such a maximal temperature is due to the increase of the number of thermally excited quasiparticles, which produce enhanced Joule heat. By lowering the temperature the cooling power at optimal bias first increases and reaches a maximum. At lower temperatures, the Joule heat due to Andreev processes causes the cooling power to decrease. At a certain temperature $T_{\min }$, the cooling power tends to zero, which defines the lower limiting temperature for the cooling regime. As follows from Fig. 7, the temperature $T_{\min }$ decreases when increasing the exchange field; this is because the Andreev current and the associated Joule heat are suppressed by the exchange 
interaction in the ferromagnet. Finally, one can see from Fig. 7 that the minimum cooling temperature in the parallel $T_{\min }^{P}$ and antiparallel $T_{\min }^{A P}$ configuration satisfy: $T_{\min }^{A P}<T_{\min }^{P}$ for $h=$ $\Delta(T)$, while $T_{\min }^{A P}>T_{\min }^{P}$ for $h=1.7 \Delta(T)$. For $h=8 \Delta(T)$ [Fig. 7(c)] $P(T)$ is almost independent on $\alpha$.

A common feature of Figs. 5, 6, and 7 is that for rather small value of the exchange field, $h=\Delta(T)$, the antiparallel configuration is more favorable for cooling [see (a) panels]. For larger exchange field $h=1.7 \Delta(T)$, on the contrary, the parallel configuration is favorable for cooling [see (b) panels]. As expected, in the case of strong enough ferromagnet $[h=8 \Delta(T)]$ the thickness of $\mathrm{F}_{1}$ layer $l_{1} \gg \xi_{h}$ and the superconducting condensate practically does not penetrate into $\mathrm{F}_{2}$ layer. Thus the cooling power is $\alpha$ independent [see Figs. 5(c), 6(c), and 7(c)].

\section{CONCLUSION}

We have developed a quantitative theory of charge and heat transport in normal metal-superconductor tunnel junctions with an intermediate ferromagnetic bilayer. We have assumed that the magnetizations of the ferromagnets form an angle $\alpha$ and focused our study on the cooling power of such a structure.

In previous works it has been suggested that the larger the exchange field the more efficient the cooling. ${ }^{18}$ In this case the enhancement of the cooling is due to the suppression of the Andreev processes and therefore suppression of the Joule heating, released in the normal metal electrode. However, our results have shown that this hypothesis is only valid in the case of strong ferromagnets $[h \gg \Delta]$. For weak ferromagnets with an exchange field comparable to the superconducting order parameter $\Delta$ the cooling power shows a nonmonotonic dependence on $h$, with a minimum at $h \approx \Delta$ (in the monodomain case) that corresponds to a maximum in the Andreev current $I_{A}$. Moreover, around this minimum the cooling power of the $\mathrm{SIF}_{1} \mathrm{~F}_{2} \mathrm{~N}$ structure is even lower than the one of the NIS junction. We have also shown that in the two-domain case, a finite value of $\alpha$ shifts the minimum of cooling power to larger values of $h$ if the thickness of $F_{1}$ is comparable to the magnetic length $\xi_{h}$. In this case, the effective exchange field $\bar{h}$ acting on the Cooper pairs is gradually reduced as $\alpha$ increases from 0 to $\pi$. The minimum then is at $\bar{h} \approx \Delta$, which corresponds to larger values of the bare $h$. Thus, for exchange fields $h \lesssim \Delta$ the antiparallel magnetic configuration ( $\alpha=\pi$ ) of magnetization leads to larger values of the cooling power. Such small exchange fields can be realized in weak ferromagnetic alloys, ${ }^{45}$ or in hybrid structures consisting of ferromagnetic insulators in contact with superconductors. ${ }^{46,47}$ For values of $h$ larger than $\Delta$, the parallel configuration $(\alpha=0)$ is the one that leads to larger values of the cooling power. For values of $h \gg \Delta$ the cooling is almost independent of $\alpha$.

Finally we have analyzed the dependence of the cooling power on the bias voltage, the tunneling parameter and the temperature. The optimized values for more efficient cooling are shown in Figs. 5, 6, and 7.

\section{ACKNOWLEDGMENTS}

The authors thank E. V. Bezuglyi, S. Kawabata, and J. P. Pekola for useful discussions. This work was supported by the Spanish Ministry of Economy and Competitiveness under Project No. FIS2011-28851-C02-02 and the Basque Government under UPV/EHU Project IT-366-07. The work of A.O. was supported by the Spanish National Research Council (CSIC) and the European Social Fund under JAE-Predoc program. A.S.V. acknowledge the hospitality of Donostia International Physics Center (DIPC), during his stay in Spain.
${ }^{1}$ M. Nahum, T. M. Eiles, and J. M. Martinis, Appl. Phys. Lett. 65, 3123 (1994).

${ }^{2}$ M. M. Leivo, J. P. Pekola, and D. V. Averin, Appl. Phys. Lett. 68, 1996 (1996).

${ }^{3}$ F. Giazotto, T. T. Heikkilä, A. Luukanen, A. M. Savin, and J. P. Pekola, Rev. Mod. Phys. 78, 217 (2006).

${ }^{4}$ P. Virtanen and T. T. Heikkilä, Appl. Phys. A 89, 625 (2007).

${ }^{5}$ J. T. Muhonen, M. Meschke, and J. P. Pekola, Rep. Prog. Phys. 75, 046501 (2012).

${ }^{6}$ A. M. Clark, N. A. Miller, A. Williams, S. T. Ruggiero, G. C. Hilton, L. R. Vale, J. A. Beall, K. D. Irwin, and J. N. Ullom, Appl. Phys. Lett. 86, 173508 (2005).

${ }^{7}$ G. C. O'Neil, P. J. Lowell, J. M. Underwood, and J. N. Ullom, Phys. Rev. B 85, 134504 (2012).

${ }^{8}$ J. P. Pekola, T. T. Heikkilä, A. M. Savin, J. T. Flyktman, F. Giazotto, and F. W. J. Hekking, Phys. Rev. Lett. 92, 056804 (2004).

${ }^{9}$ A. S. Vasenko and F. W. J. Hekking, J. Low Temp. Phys. 154, 221 (2009); S. Rajauria, H. Courtois, and B. Pannetier, Phys. Rev. B 80, 214521 (2009).

${ }^{10}$ B. Jug and Z. Trontelj, IEEE Trans. Appl. Supercond. 11, 848 (2001); J. Phys.: Conf. Ser. 97, 012097 (2008).
${ }^{11}$ J. P. Pekola, D. V. Anghel, T. I. Suppula, J. K. Suoknuuti, A. J. Manninen, and M. Manninen, Appl. Phys. Lett. 76, 2782 (2000); D. Golubev and A. Vasenko, in International Workshop on Superconducting Nano-electronics Devices, edited by J. Pekola, B. Ruggiero, and P. Silvestrini (Kluwer Academic, Dordrecht, 2002), p. 165 .

${ }^{12}$ J. T. Peltonen, J. T. Muhonen, M. Meschke, N. B. Kopnin, and J. P. Pekola, Phys. Rev. B 84, 220502(R) (2011).

${ }^{13}$ S. Chaudhuri and I. J. Maasilta, Phys. Rev. B 85, 014519 (2012).

${ }^{14}$ A. F. Andreev, Zh. Eksp. Teor. Fiz. 46, 1823 (1964) [Sov. Phys. JETP 19, 1228 (1964)]; D. Saint-James, J. Phys. (Paris) 25, 899 (1964).

${ }^{15}$ S. Rajauria, P. Gandit, T. Fournier, F. W. J. Hekking, B. Pannetier, and H. Courtois, Phys. Rev. Lett. 100, 207002 (2008); J. Low Temp. Phys. 154, 211 (2009).

${ }^{16}$ A. Bardas and D. Averin, Phys. Rev. B 52, 12873 (1995).

${ }^{17}$ A. S. Vasenko, E. V. Bezuglyi, H. Courtois, and F. W. J. Hekking, Phys. Rev. B 81, 094513 (2010).

${ }^{18}$ F. Giazotto, F. Taddei, R. Fazio, and F. Beltram, Appl. Phys. Lett. 80, 3784 (2002). 
${ }^{19}$ M. J. M. de Jong and C. W. J. Beenakker, Phys. Rev. Lett. 74, 1657 (1995).

${ }^{20}$ A. A. Golubov, M. Yu. Kupriyanov, and E. Il'ichev, Rev. Mod. Phys. 76, 411 (2004).

${ }^{21}$ A. I. Buzdin, Rev. Mod. Phys. 77, 935 (2005).

${ }^{22}$ F. S. Bergeret, A. F. Volkov, and K. B. Efetov, Rev. Mod. Phys. 77, 1321 (2005)

${ }^{23}$ F. Giazotto, F. Taddei, M. Governale, C. Castellana, R. Fazio, and F. Beltram, Phys. Rev. Lett. 97, 197001 (2006).

${ }^{24}$ A. V. Burmistrova, I. A. Devyatov, M. Yu. Kupriyanov, and T. Yu. Karminskaya, JETP Lett. 93, 203 (2011).

${ }^{25}$ S. Oh, D. Youm, and M. R. Beasley, Appl. Phys. Lett. 71, 2376 (1997); Ya. V. Fominov, A. A. Golubov, T. Yu. Karminskaya, M. Yu. Kupriyanov, R. G. Deminov, and L. R. Tagirov, JETP Lett. 91, 308 (2010).

${ }^{26}$ K. Yu. Arutyunov, H.-P. Auraneva, and A. S. Vasenko, Phys. Rev. B 83, 104509 (2011).

${ }^{27}$ A. I. Larkin and Yu. N. Ovchinnikov, in Nonequilibrium Superconductivity, edited by D. N. Langenberg and A. I. Larkin (Elsevier, Amsterdam, 1986).

${ }^{28}$ W. Belzig, F. K. Wilhelm, C. Bruder, G. Schön, and A. D. Zaikin, Superlattices Microstruct. 25, 1251 (1999).

${ }^{29}$ K. D. Usadel, Phys. Rev. Lett. 25, 507 (1970).

${ }^{30}$ D. A. Ivanov and Ya. V. Fominov, Phys. Rev. B 73, 214524 (2006).

${ }^{31}$ F. S. Bergeret, A. F. Volkov, and K. B. Efetov, Phys. Rev. B 66, 184403 (2002).

${ }^{32}$ M. Yu. Kuprianov and V. F. Lukichev, Zh. Eksp. Teor. Fiz. 94, 139 (1988) [Sov. Phys. JETP 67, 1163 (1988)].
${ }^{33}$ E. V. Bezuglyi, A. S. Vasenko, V. S. Shumeiko, and G. Wendin, Phys. Rev. B 72, 014501 (2005); E. V. Bezuglyi, A. S. Vasenko, E. N. Bratus, V. S. Shumeiko, and G. Wendin, ibid. 73, 220506(R) (2006).

${ }^{34}$ F. S. Bergeret, A. F. Volkov, and K. B. Efetov, Phys. Rev. Lett. 86, 4096 (2001).

${ }^{35}$ R. S. Keizer, S. T. B. Goennenwein, T. M. Klapwijk, G. Miao, G. Xiao, and A. Gupta, Nature (London) 439, 825 (2006).

${ }^{36}$ G. B. Halász, J. W. A. Robinson, J. F. Annett, and M. G. Blamire, Phys. Rev. B 79, 224505 (2009).

${ }^{37}$ T. S. Khaire, M. A. Khasawneh, W. P. Pratt Jr., and N. O. Birge, Phys. Rev. Lett. 104, 137002 (2010).

${ }^{38}$ J. W. A. Robinson, J. D. S. Witt, and M. G. Blamire, Science 329, 59 (2010).

${ }^{39}$ E. V. Bezuglyi and V. Vinokur, Phys. Rev. Lett. 91, 137002 (2003).

${ }^{40}$ T. Yokoyama, Y. Tanaka, A. A. Golubov, and Y. Asano, Phys. Rev. B 72, 214513 (2005).

${ }^{41}$ N. R. Werthamer, Phys. Rev. 147, 255 (1966).

${ }^{42}$ We neglect the contribution to the Andreev current due to the partial Andreev reflection at the energies above the superconducting gap. In the case of strong enough tunnel barriers, this contribution leads to a small correction and can be neglected (see Ref. 17 for details).

${ }^{43}$ F. S. Bergeret, A. F. Volkov, and K. B. Efetov, Phys. Rev. Lett. 86, 3140 (2001).

${ }^{44}$ A. Ozaeta, A. S. Vasenko, F. W. J. Hekking, and F. S. Bergeret, e-print arXiv:1203.3679.

${ }^{45}$ T. Kontos, M. Aprili, J. Lesueur, X. Grison, and L. Dumoulin, Phys. Rev. Lett. 93, 137001 (2004).

${ }^{46}$ T. Tokuyasu, J. A. Sauls, and D. Rainer, Phys. Rev. B 38, 8823 (1988).

${ }^{47}$ A. Cottet, Phys. Rev. Lett. 107, 177001 (2011). 